\title{
Triangulations of Cayley and Tutte polytopes
}

\author{
Matjaž Konvalinka ${ }^{1}$ and Igor Pak $^{2}$ \\ ${ }^{1}$ Department of Mathematics, University of Ljubljana, Slovenia \\ ${ }^{2}$ Department of Mathematics, UCLA, Los Angeles, California, USA
}

\begin{abstract}
Cayley polytopes were defined recently as convex hulls of Cayley compositions introduced by Cayley in 1857. In this paper we resolve Braun's conjecture, which expresses the volume of Cayley polytopes in terms of the number of connected graphs. We extend this result to a two-variable deformations, which we call Tutte polytopes. The volume of the latter is given via an evaluation of the Tutte polynomial of the complete graph.

Our approach is based on an explicit triangulation of the Cayley and Tutte polytope. We prove that simplices in the triangulations correspond to labeled trees and forests. The heart of the proof is a direct bijection based on the neighbors-first search graph traversal algorithm.

Résumé. Les polytopes de Cayley ont été définis récemment comme des ensembles convexes de compositions de Cayley introduits par Cayley en 1857. Dans ce papier, nous résolvons la conjecture de Braun. Cette dernière exprime le volume du polytopes de Cayley en termes du nombre de graphes connexes. Nous étendons ce résultat à des déformations de polytopes de Cayley à deux variables, à savoir les polytopes de Tutte. Le volume de ces derniers est donné par une évaluation du polynôme de Tutte du graphe complet.

Notre approche est basée sur une triangulation explicite des polytopes de Cayley et Tutte. Nous démontrons que les simplexes de ces triangulations correspondent à des arbres marqués. La pierre angulaire de notre démonstration est une bijection directe basées sur l'algorithme de la recherche du premier voisin sur le graphe.
\end{abstract}

Keywords: polytopes, volume, Cayley compositions, Tutte polynomial

\section{Introduction}

In the past several decades, there has been an explosion in the number of connections and applications between geometric and enumerative combinatorics. Among those, a number of new families of "combinatorial polytopes" were discovered, whose volume has a combinatorial significance. Still, whenever a new family of $n$-dimensional polytopes is discovered whose volume is a familiar integer sequence (up to scaling), it feels like a "minor miracle", a familiar face in a crowd in a foreign country, a natural phenomenon in need of an explanation.

In this extended abstract we prove a surprising conjecture due to Ben Braun $[\overline{\mathrm{BBL}}]$, which expresses the volume of the Cayley polytope in terms of the number of connected labeled graphs. Our proof is robust enough to allow generalizations in several directions, leading to the definition of Tutte polytopes, and largely explaining this latest "minor miracle".

We start with the following classical result. 
Theorem 1 (Cayley, 1857) The number of integer sequences $\left(a_{1}, \ldots, a_{n}\right)$ such that $1 \leq a_{1} \leq 2$, and $1 \leq$ $a_{i+1} \leq 2 a_{i}$ for $1 \leq i<n$, is equal to the total number of partitions of integers $N \in\left\{0,1, \ldots, 2^{n}-1\right\}$ into parts $1,2,4, \ldots, 2^{n-1}$.

Although Cayley's original proof [Cay] uses only elementary generating functions, it inspired a number of other proofs and variations [APRS, BBL, CLS, KP2]. It turns out that Cayley's theorem is best understood in a geometric setting, as an enumerative problem for the number of integer points in an $n$-dimensional polytope defined by the inequalities as in the theorem.

Following [BBL], define the Cayley polytope $\mathbf{C}_{n} \subset \mathbb{R}^{n}$ by inequalities:

$$
1 \leq x_{1} \leq 2, \text { and } 1 \leq x_{i} \leq 2 x_{i-1} \text { for } i=2, \ldots, n,
$$

so that the number of integer points in $\mathbf{C}_{n}$ is the number of integer sequences $\left(a_{1}, \ldots, a_{n}\right)$, and the number of certain partitions, as in Cayley's theorem.

The polytope $\mathbf{C}_{2}$ is the trapezoid with bold boundaries in Figure 1 with $t=1$. The polytope $\mathbf{C}_{3}$ is shown from two different angles on the right-hand side of Figure 3 Note the volume of these polytopes is 2 and $19 / 3$, respectively.

In [BBL], Braun made the following interesting conjecture about the volume of $\mathbf{C}_{n}$. Denote by $\mathcal{C}_{n}$ the set of connected graphs (with no loops or multiple edges) on $n$ nodes. (To avoid ambiguity, throughout the paper, we distinguish graph nodes from polytope vertices.) Let $C_{n}=\left|\mathcal{C}_{n}\right|$.

Theorem 2 (Formerly Braun's conjecture) Let $\mathbf{C}_{n} \subset \mathbb{R}^{n}$ be the Cayley polytope defined above. Then $\operatorname{vol} \mathbf{C}_{n}=C_{n+1} / n$ !

This is the first in a long chain of results we present in [KP1], leading to the following general result. Let $0<q \leq 1$ and $t \geq 0$. Define the Tutte polytope $\mathbf{T}_{n}(q, t) \subset \mathbb{R}^{n}$ by inequalities: $x_{n} \geq 1-q$ and

$$
q x_{i} \leq q(1+t) x_{i-1}-t(1-q)\left(1-x_{j-1}\right),
$$

where $1 \leq j \leq i \leq n$ and $x_{0}=1$. Note that for $j=1$ and $i>1$, the inequality is $x_{i} \leq(1+t) x_{i-1}$, while for $i=j=1$, it is $x_{i} \leq 1+t$.

Theorem 3 (Main result) Let $\mathbf{T}_{n}(q, t) \subset \mathbb{R}^{n}$ be the Tutte polytope defined above. Then

$$
\operatorname{vol} \mathbf{T}_{n}(q, t)=t^{n} \mathcal{T}_{K_{n+1}}(1+q / t, 1+t) / n !,
$$

where $\mathcal{T}_{H}(x, y)$ denotes the Tutte polynomial of the graph $H$.

One can show that in certain sense, Tutte polytopes are a two variable deformation of the Cayley polytope:

$$
\lim _{q \rightarrow 0+} \mathbf{T}_{n}(q, 1)=\mathbf{C}_{n} .
$$

To see this, note that for $t=1$, the inequalities with $j=1$ in (1) give $x_{i} \leq 2 x_{i-1}$, and for $j>1$, we get $x_{j-1} \geq 1$ as $q \rightarrow 0+$.

Now, recall that $\mathcal{T}_{H}(1,2)$ is the number of connected subgraphs of $H$, a standard property of Tutte polynomials (see e.g. [Bol]). Letting $q \rightarrow 0+$ and $t=1$ shows that Theorem 2 follows immediately from 
Theorem 3. In other words, our main theorem is an advanced generalization of Braun's Conjecture (now Theorem 2 .

The proof of both Theorem 2 and 3 is based on explicit triangulations of polytopes. The simplices in the triangulations have a combinatorial nature, and are in bijection with labeled trees (for the Cayley polytope) and forests (for the Tutte polytope) on $n+1$ nodes. This bijection is based on a variant of the neighbors-first search (NFS) graph traversal algorithm studied by Gessel and Sagan [GS]. Roughly speaking, in the case of Cayley polytopes, the volume of a simplex in bijection with a labeled tree $T$ corresponds to the set of labeled graphs for which $T$ is the output of the NFS.

Rather than elaborate on the inner working of the proof, we illustrate the idea in the following example.

Example 4 The triangulation of $\mathcal{T}_{2}(q, t)$ is shown in Figure 1 For example, the top triangle is labeled by the tree with edges 12 and 13 ; its area, multiplied by 2 !, is $t^{2}(1+t)$, and it also has two graphs that map into it, the tree itself (with two edges) and the complete graph on 3 nodes (with three edges).

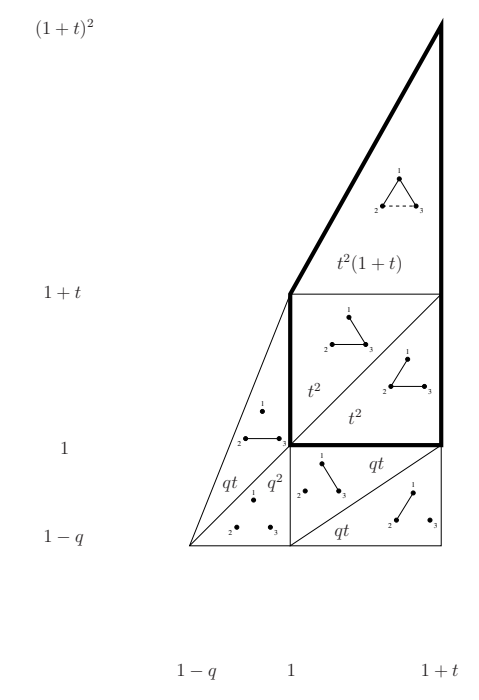

Fig. 1: A triangulation of the Tutte polytope $\mathbf{T}_{2}(q, t)$.

The rest of the extended abstract is structured as follows. We begin with definitions and basic combinatorial results in Section 2. In Sections 3 we construct a triangulation of the Cayley polytope. Tutte polytopes are analyzed in Section 4 . We conclude with final remarks in Section 5. The reader should consult [KP1] for details.

\section{Combinatorial and geometric preliminaries}

A labeled tree is a connected acyclic graph. We take each labeled tree to be rooted at the node with the maximal label. A labeled forest is an acyclic graph. Its components are labeled trees, and we root each of them at the node with the maximal label. An unlabeled plane forest is a graph without cycles in which 
we do not distinguish the nodes, but we choose a root in each component, which is an unlabeled plane tree, and the subtrees at any node, as well as the components of the graph, are linearly ordered (from left to right).

For a (multi)graph $G$ on the set of nodes $V$, denote by $k(G)$ the number of connected components of $G$, and by $e(G)$ the number of edges of $G$. Consider the polynomial

$$
\mathcal{Z}_{G}(q, t)=\sum_{H \subseteq G} q^{k(H)-k(G)} t^{e(H)},
$$

where the sum is over all spanning subgraphs $H$ of $G$. This polynomial is a statistical sum in the random cluster model in statistical mechanics. It is related to the Tutte polynomial

$$
\mathcal{T}_{G}(x, y)=\sum_{H \subseteq G}(x-1)^{k(H)-k(G)}(y-1)^{e(H)-|V|+k(H)}
$$

by the equation

$$
\mathcal{T}_{G}(x, y)=(y-1)^{k(G)-|V|} \mathcal{Z}_{G}((x-1)(y-1), y-1) .
$$

Tutte's classical result is a combinatorial interpretation of the coefficients of the Tutte polynomial [Tut]. He showed that for a connected graph $G$ we have $\mathcal{T}(x, y)=\sum_{T} x^{\mathrm{ia}(T)} y^{\operatorname{ea}(T)}$, where the summation is over all spanning trees $T$ in $G$; here $\mathrm{ia}(T)$ and ea $(T)$ denote the number of internally active and externally active edges in $T$, respectively. While both ia $(T)$ and ea $(T)$ depend on the ordering of the edges in $G$, the sum on the right does not (see [Bol, $\S$ X.5] for definitions and details).

For the complete graph $K_{n}$, the Tutte polynomial and its evaluations are well studied (see [Tut, Ges2]). In this case, under a lexicographic ordering of edges, the statistics ia $(T)$ and ea $(T)$ can be interpreted combinatorially [Ges2, GS] via the neighbor-first search (NFS) introduced in [GS], a variant of which is also crucial for our purposes. Take a labeled connected graph $G$ on $n+1$ nodes. Choose the node with the maximal label, i.e. $n+1$, as the first active node (and also the 0-th visited node). At each step, visit the previously unvisited neighbors of the active node in decreasing order of their labels, and make the one with the smallest label the new active node. Note that in [GS], the NFS starts at the node with the minimal label, and the neighbors of the active node are visited in increasing order of their labels. If all the neighbors of the active node have been visited, backtrack to the last visited node that has not been an active node, and make it the new active node. The resulting search tree $T$ is a labeled tree on $n+1$ nodes, we denote it $\Phi(G)$ (see Example 5 .

Example 5 Let $G$ be the graph on the left-hand side of Figure2. The neighbors-first search starts in node 12 and visits the other nodes in order $11,10,6,8,7,9,3,1,4,2,5$. The resulting search tree $\Phi(G)$ is on the right-hand side of Figure 2 The edges of $G$ that are not in $\Phi(G)$ are dashed.

In a special case, the polynomial $\operatorname{Inv}_{n}(y)=\mathcal{T}_{K_{n}}(1, y) y^{1-n}$ is the classical inversion polynomial [MR] (see also [Ges1, GW, GouJ]), a generating function for the number of spanning trees with respect to inversions.

\section{A triangulation of the Cayley polytope}

Attach a coordinate of the form $x_{i} / 2^{j}$ to each node of the tree $T$ rooted at the node with label $n+1$, where $i$ is the position of the node in the NFS, and $j$ is a non-negative integer defined as follows. Attach 


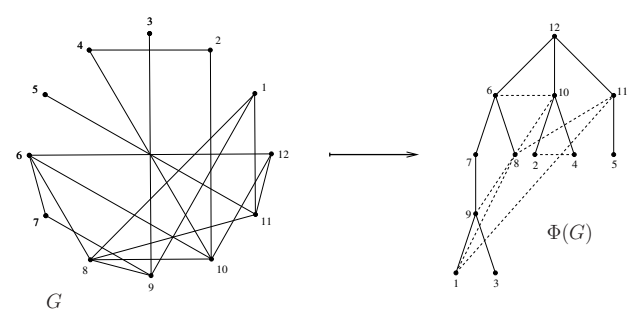

Fig. 2: A connected graph and its NFS tree.

$x_{0}=1$ to the root; and if the node $v$ has coordinate $x_{i} / 2^{j}$ and successors $v_{1}, \ldots, v_{k}$ (in increasing order of their labels), then make the coordinates of $v_{k}, \ldots, v_{1}$ to be $x_{i^{\prime}} / 2^{j}, x_{i^{\prime}+1} / 2^{j+1}, \ldots, x_{i^{\prime}+k-1} / 2^{j+k-1}$. See Figure 3 left, for an example.
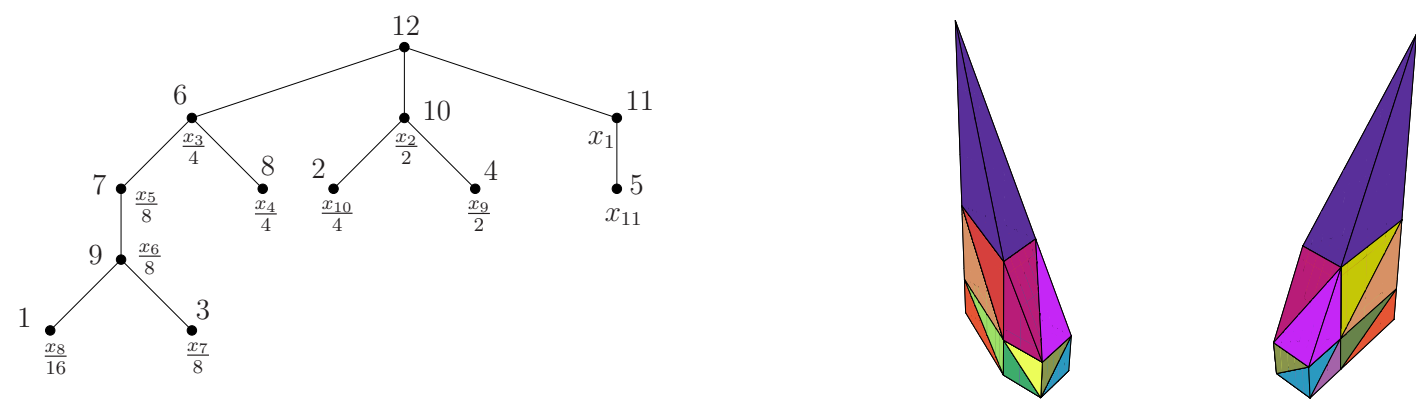

Fig. 3: Coordinates in a tree. A triangulation of $\mathbf{C}_{3}$ from two different angles.

Define $\alpha(T)=\sum_{i} j_{i}$. For the next lemma, which gives another characterization of $\alpha(T)$, note first that in a rooted labeled tree (as well as in a plane tree), we have the natural concept of an up (respectively, down) step, i.e. a step from a node to its parent (respectively, from a node to its child), as well as a down right step, i.e. a down step $v \rightarrow v^{\prime \prime}$ that follows an up step $v^{\prime} \rightarrow v$ so that $v^{\prime \prime}$ has a larger label than (or is the the right of) $v^{\prime}$. Call a path of length $k \geq 2$ in a rooted labeled tree (or a plane tree) a cane path if the first $k-1$ steps are up and the last one is down right. Some cane paths in the tree from Figure 3 are from 9 to 8 , from 3 to 11 , and from 2 to 4 .

Lemma 6 For a node $v$ with coordinate $x_{i} / 2^{j}, j$ is the number of cane paths in $T$ that start in $v$. In particular, $\alpha(T)$ is the number of cane paths in $T$.

Arrange the coordinates of the nodes $1, \ldots, n$ according to the labels. More precisely, define

$$
\mathbf{S}_{T}=\left\{\left(x_{1}, \ldots, x_{n}\right): 1 \leq x_{i_{1}} / 2^{j_{1}} \leq x_{i_{2}} / 2^{j_{2}} \leq \ldots \leq x_{i_{n}} / 2^{j_{n}} \leq 2\right\},
$$

where the coordinate of the node with label $k$ is $x_{i_{k}} / 2^{j_{k}}$. For the tree from Figure 3 , the corresponding inequalities are

$$
1 \leq \frac{x_{8}}{16} \leq \frac{x_{10}}{4} \leq \frac{x_{7}}{8} \leq \frac{x_{9}}{2} \leq x_{11} \leq \frac{x_{3}}{4} \leq \frac{x_{5}}{8} \leq \frac{x_{4}}{4} \leq \frac{x_{6}}{8} \leq \frac{x_{2}}{2} \leq x_{1} \leq 2 .
$$


Theorem 7 For every labeled tree $T$ on $n+1$ nodes, the set $\mathbf{S}_{T}$ is a simplex, and

$$
n ! \operatorname{vol} \mathbf{S}_{T}=\mid\left\{G \in \mathcal{C}_{n+1}, \text { s.t. } \Phi(G)=T\right\} \mid=2^{\alpha(T)}
$$

Furthermore, simplices $\mathbf{S}_{T}$ triangulate the Cayley polytope $\mathbf{C}_{n}$. In particular, $n ! \operatorname{vol} \mathbf{C}_{n}=\left|\mathcal{C}_{n+1}\right|$.

Figure 3, right, shows two views of the resulting triangulation of $\mathbf{C}_{3}$. Note that the theorem implies Braun's conjecture (Theorem 2). What follows is a brief sketch of the proof of Theorem 7

Erase all labels (not the coordinates) from the labeled tree $\Phi(G)$, to make it into a plane tree $\Psi(G)$. For each node $v$ of a plane tree $T$ with successors with coordinates $x_{i} / 2^{j}, x_{i+1} / 2^{j+1}, \ldots, x_{i+k-1} / 2^{j+k-1}$, take inequalities $1 \leq x_{i+k-1} / 2^{j+k-1} \leq \ldots \leq x_{i+1} / 2^{j+1} \leq x_{i} / 2^{j} \leq 2$. Equivalently, take inequalities

$$
\begin{aligned}
2^{j} \leq & x_{i} \leq 2^{j+1} \\
2^{j+1} \leq & x_{i+1} \leq 2 x_{i} \\
& \vdots \\
2^{j+k-1} \leq & x_{i+k-1} \leq 2 x_{i+k-2} .
\end{aligned}
$$

Denote the resulting polytope $\mathbf{D}_{T}$. It is easy to see that $\mathbf{D}_{T}$ is triangularized by simplices $\mathbf{S}_{T^{\prime}}$, where $T^{\prime}$ runs over labeled trees whose underlying plane tree is $T$. We have to prove that $\left\{\mathbf{D}_{T}: T\right.$ a plane tree $\}$ is a subdivision of $\mathbf{C}_{n}$.

The Cayley polytope $\mathbf{C}_{n}$ consists of all points $\left(x_{1}, \ldots, x_{n}\right)$ for which $1 \leq x_{1} \leq 2$ and $1 \leq x_{i} \leq 2 x_{i-1}$ for $i=2, \ldots, n$. The main idea of the proof of Theorem 7 is to divide these inequalities into "narrower" inequalities. We state this precisely in the following example, and then in full generality.

Example 8 Since $1 \leq x_{2} \leq 2 x_{1}$ and $2 x_{1} \geq 2$, we have either $1 \leq x_{2} \leq 2$ or $2 \leq x_{2} \leq 2 x_{1}$. If

\begin{tabular}{|c|c|c|c|}
\hline \multirow[t]{14}{*}{$1 \leq x_{1} \leq 2$} & \multirow[t]{5}{*}{$1 \leq x_{2} \leq 2$} & \multirow[t]{2}{*}{$1 \leq x_{3} \leq 2$} & $1 \leq x_{4} \leq 2$ \\
\hline & & & $2 \leq x_{4} \leq 2 x_{3}$ \\
\hline & & \multirow[t]{3}{*}{$2 \leq x_{3} \leq 2 x_{2}$} & $1 \leq x_{4} \leq 2$ \\
\hline & & & $2 \leq x_{4} \leq 4$ \\
\hline & & & $4 \leq x_{4} \leq 2 x_{3}$ \\
\hline & \multirow{9}{*}{$2 \leq x_{2} \leq 2 x_{1}$} & \multirow[t]{2}{*}{$1 \leq x_{3} \leq 2$} & $1 \leq x_{4} \leq 2$ \\
\hline & & & $2 \leq x_{4} \leq 2 x_{3}$ \\
\hline & & \multirow[t]{3}{*}{$2 \leq x_{3} \leq 4$} & $1 \leq x_{4} \leq 2$ \\
\hline & & & $2 \leq x_{4} \leq 4$ \\
\hline & & & $4 \leq x_{4} \leq 2 x_{3}$ \\
\hline & & \multirow[t]{4}{*}{$4 \leq x_{3} \leq 2 x_{2}$} & $1 \leq x_{4} \leq 2$ \\
\hline & & & $2 \leq x_{4} \leq 4$ \\
\hline & & & $4 \leq x_{4} \leq 8$ \\
\hline & & & $8 \leq x_{4} \leq 2 x_{3}$ \\
\hline
\end{tabular}
$1 \leq x_{2} \leq 2$, then either $1 \leq x_{3} \leq 2$ or $2 \leq x_{3} \leq 2 x_{2}$. On the other hand, if $2 \leq x_{2} \leq 2 x_{1}$, then $2 x_{2} \geq 4$, so we have $1 \leq x_{3} \leq 2,2 \leq x_{3} \leq 4$ or $4 \leq x_{3} \leq 2 x_{2}$. The following table presents all such choices for $n=4$.

Lemma 9 The Cayley polytope $\mathbf{C}_{n}$ can be subdivided into polytopes defined by inequalities for variables $x_{1}, \ldots, x_{n}$ so that: the inequalities for $x_{1}$ are $1 \leq x_{1} \leq 2$; the inequalities for each $x_{i}$ are either $2^{j_{i}} \leq x_{i} \leq 2^{j_{i}+1}$ or $2^{j_{i}} \leq x_{i} \leq 2 x_{i-1}$ (only if $i \geq 2$ ) for some $j_{i} \geq 0$; for $i \geq 2$, we have $j_{i} \leq j_{i-1}+1$, and $j_{i}=j_{i-1}+1$ if and only if the inequalities for $x_{i}$ are $2^{j_{i}} \leq x_{i} \leq 2 x_{i-1}$. 
We claim the the polytopes constructed in the lemma are precisely the polytopes $\mathbf{D}_{T}$. So say that we have inequalities satisfying the conditions of the lemma and defining a polytope $\mathbf{P}$. Our goal is to construct the unique plane tree $T$ satisfying $\mathbf{D}_{T}=\mathbf{P}$.

Assume that $k, 1 \leq k \leq n$, is the largest integer so that the inequalities for $x_{i}, i=2, \ldots, k$, are of the form $2^{i-1} \leq x_{i} \leq 2 x_{i-1}$. In particular, the inequalities for $x_{k+1}$ are not of the form $2^{k} \leq x_{k+1} \leq 2 x_{k}$, but instead $2^{j_{k+1}} \leq x_{k+1} \leq 2^{j_{k+1}+1}$ for some $j_{k+1}, 0 \leq j_{k+1} \leq k-1$.

There exist unique integers $a_{1}, \ldots, a_{k} \geq 1, a_{1}+\ldots+a_{k}=n-k$, satisfying the following properties:

$$
\begin{aligned}
j_{k+1}, \ldots, j_{k+a_{1}} & \geq k-1, & j_{k+a_{1}+1} & <k-1 \\
j_{k+a_{1}+1}, \ldots, j_{k+a_{1}+a_{2}} & \geq k-2, & j_{k+a_{1}+a_{2}+1} & <k-2 \\
j_{k+a_{1}+a_{2}+1}, \ldots, j_{k+a_{1}+a_{2}+a_{3}} & \geq k-3, & j_{a_{1}+a_{2}+a_{3}+1} & <k-3
\end{aligned}
$$

Note that if $a_{1} \geq 1$, then $j_{k+1}=k-1$, if $a_{2} \geq 1$, then $j_{k+a_{1}+1}=k-2$, etc.

In other words, among the inequalities for $x_{k+1}, \ldots, x_{n}$, the first $a_{1}$ inequalities have at least $2^{k-1}$ on the left, the next $a_{2}$ inequalities have at least $2^{k-2}$ on the left, etc. Say that among the inequalities for $x_{k+1}, \ldots, x_{n}$, the first $a_{1}$ inequalities define the polytope $2^{k-1} \mathbf{P}_{1}$, the next $a_{2}$ inequalities define the polytope $2^{k-2} \mathbf{P}_{2}$, etc. By induction, the polytopes $\mathbf{P}_{1}, \ldots, \mathbf{P}_{k}$ are of the form $\mathbf{D}_{T_{1}}, \ldots, \mathbf{D}_{T_{k}}$ for some plane trees $T_{1}, \ldots, T_{k}$ on $a_{1}+1, a_{2}+1, \ldots, a_{k}+1$ nodes. Define the tree $T$ by taking a root with $k$ successors and subtrees $T_{1}, \ldots, T_{k}$. One can check that indeed $\mathbf{D}_{T}=\mathbf{P}$.

Example 10 Say that

$$
\mathbf{P}=\left\{\begin{array}{lll}
\left(x_{1}, \ldots, x_{11}\right): & 1 \leq x_{1} \leq 2, & 2 \leq x_{2} \leq 2 x_{1} \\
4 \leq x_{3} \leq 2 x_{2}, & 4 \leq x_{4} \leq 8, & 8 \leq x_{5} \leq 2 x_{4} \\
8 \leq x_{6} \leq 16, & 8 \leq x_{7} \leq 16, & 16 \leq x_{8} \leq 2 x_{7} \\
2 \leq x_{9} \leq 4, & 4 \leq x_{10} \leq 2 x_{9}, & 1 \leq x_{11} \leq 2
\end{array}\right\}
$$

We have $k=3$ and $a_{1}=5, a_{2}=2, a_{3}=1$. Furthermore,

$$
\begin{aligned}
& \mathbf{P}_{1}=\left\{\begin{array}{lll}
\left(x_{1}, \ldots, x_{5}\right): & 1 \leq x_{1} \leq 2, & 2 \leq x_{2} \leq 2 x_{1}, \\
2 \leq x_{3} \leq 4, & 2 \leq x_{4} \leq 4, & 4 \leq x_{5} \leq 2 x_{4}
\end{array}\right\}, \\
& \mathbf{P}_{2}=\left\{\left(x_{1}, x_{2}\right): \quad 1 \leq x_{1} \leq 2, \quad 2 \leq x_{2} \leq 2 x_{1}\right\}, \\
& \mathbf{P}_{3}=\left\{x_{1}: \quad 1 \leq x_{1} \leq 2\right\} \text {. }
\end{aligned}
$$

The corresponding subtrees $T_{1}, T_{2}, T_{3}$ of the tree $T$ is shown with full lines in Figure 4

\section{A triangulation of the Tutte polytope}

In this section, we sketch the construction of a triangulation of the Tutte polytope that proves Theorem 3 . Say we are given a labeled forest $F$. Order the components so that the maximal labels in the components are decreasing. If a node $v$ has the maximal label in its component and there are $i$ nodes in previous 


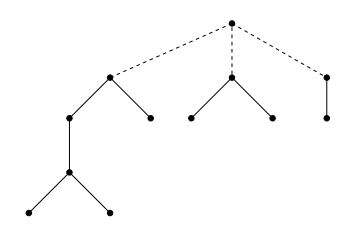

Fig. 4: The plane tree corresponding to a subpolytope.

components, choose the coordinate of $v$ to be $t\left(x_{i}-1+q\right)$. In particular, the coordinate of the node with label $n+1$ is $t\left(x_{0}-1+q\right)=q t$. Every other node $v$ has a coordinate of the form

$$
\frac{q x_{i}-(1-q)\left(1-x_{l}\right)}{(1+t)^{j}}-\left(x_{l}-1+q\right),
$$

where $i$ is the position of $v$ in NFS, $j$ is the number of cane paths in $F$ starting in $v$, and $x_{l}$ is the coordinate of the vertex with the maximal label in the component of $v$. Denote the coordinate of the node with label $k$ in a forest $F$ by $c(k, F ; t)$.

Define

$$
\mathbf{S}_{F}(q, t)=\left\{\left(x_{1}, \ldots, x_{n}\right): 0 \leq c(1, F ; q, t) \leq c(2, F ; q, t) \leq \ldots \leq c(n+1, F ; q, t)=q t\right\} .
$$

Denote by $\mathcal{G}_{n}$ the set of all (not necessarily connected) graphs on $n$ vertices. Order the components so that the maximal labels in the components are decreasing. Perform the NFS on each component of $G$ (see Section 22. The result is a labeled forest, we denote it by $\Phi(G)=F$.

Theorem 11 For every labeled forest $F$ on $n+1$ nodes, the set $\mathbf{S}_{F}(q, t)$ is a simplex, and

$$
n ! \operatorname{vol} \mathbf{S}_{F}(q, t)=\sum_{G \in \mathcal{G}_{n+1}, \Phi(G)=F} q^{k(G)-1} t^{|E(G)|}=q^{k(F)-1} t^{|E(F)|}(1+t)^{\alpha(F)} .
$$

Furthermore, simplices $\mathbf{S}_{F}(q, t)$ triangulate the Tutte polytope $\mathbf{T}_{n}(q, t)$. In particular,

$$
n ! \operatorname{vol} \mathbf{T}_{n}(q, t)=\sum_{G \in \mathcal{G}_{n+1}} q^{k(G)-1} t^{|E(G)|}=\mathcal{Z}_{K_{n+1}}(q, t)
$$

This implies Theorem 3

\section{Final remarks}

\section{1}

It might seem rather mysterious how we got from the intuitive construction of Section 3 to the complicated definition of simplices in Section 4 Indeed, as mentioned in the Introduction, Theorems 2 and 3 are just the first and the final step in a series of constructions that include the Gayley polyope, the $t$-Cayley polytope and the t-Gayley polytope. Consult [KP1] for all these constructions, as well as for omitted proofs and some applications. See [KP2] for an application to the enumeration of labeled connected graphs. 
Among these omitted results, let us just mention the one concerning vertices of the the Tutte polytope. It is almost immediate from the definition that the set of vertices of $\mathbf{C}_{n}$ is the set

$$
V_{n}=\left\{\left(x_{1}, \ldots, x_{n}\right): x_{1} \in\{1,2\}, x_{i} \in\left\{1,2 x_{i-1}\right\} \text { for } i=2, \ldots, n\right\} .
$$

Let $V_{n}(q, t)$ be the set $V_{n}(t)$ in which we replace every power of 2 by the same power of $1+t$, and the trailing 1's of each point by $1-q$. It is much less obvious that the set of vertices of $\mathbf{T}_{n}(q, t)$ is precisely $V_{n}(q, t)$. For example, the coordinates of the vertices of $\mathbf{T}_{3}(q, t)$ are given by lines in the following table:

$$
\begin{array}{ccc}
1+t & (1+t)^{2} & (1+t)^{3} \\
1+t & (1+t)^{2} & 1-q \\
1+t & 1 & 1+t \\
1+t & 1-q & 1-q \\
1 & 1+t & (1+t)^{2} \\
1 & 1+t & 1-q \\
1 & 1 & 1+t \\
1-q & 1-q & 1-q
\end{array}
$$

\section{2}

By now, there are quite a few papers on "combinatorial volumes", i.e. expressing combinatorial sequences as volumes of certain polytopes. These include Euler numbers as volumes of hypersimplices [Sta1] (see also [ABD, ERS, [LP, $\overline{\text { Pos]}})$, Catalan numbers [GGP], Cayley numbers as volumes of permutohedra (see [Pak, Zie]), the number of linear extensions of posets [Sta2], etc.

Let us mention a mysterious connection of our results to those in [SP], where the number of (generalized) parking functions appears as the volume of a certain polytope, which is also combinatorially equivalent to an $n$-cube. The authors observe that in a certain special case, their polytopes have (scaled) volume the inversion polynomial $\operatorname{Inv}_{n}(t)$, compared to $t^{n} \operatorname{Inv}_{n}(1+t)$ for the $t$-Cayley polytopes. The connection between these two families of polytopes is yet to be understood, and the authors intend to pursue this in the future.

In this connection, it is worth noting that Theorem 3 and our triangulation construction seem to be fundamentally about labeled trees rather than parking functions, since the full Tutte polynomial $\mathbf{T}_{K_{n}}(q, t)$ seems to have no known combinatorial interpretation in the context of parking functions (cf. [Sta3, $\mathrm{Hag}]$ ). Curiously, the specialization $\mathbf{T}_{G}(1, t)$ has a natural combinatorial interpretation for $G$-parking functions for general graphs [CL].

\section{3}

It is worth noting that all simplices in the triangulation of the Cayley polytopes are Schläfli orthoschemes (simplices with orthogonal sides), which play an important role in combinatorial geometry. For example, in McMullen's polytope algebra (which formalizes properties of scissor congruence), orthoschemes form a linear basis $[\mathrm{McM}]$ (see also [Dup, Pak]). Moreover, Hadwiger's conjecture states that every convex polytope in $\mathbb{R}^{d}$ can be triangulated into a finite number of orthoschemes [Had] (see also [BKKS]).

Let us emphasize here that not all simplices of triangulations constructed in Section 4 are orthoschemes. Let us also mention that triangulations of polytopes $\mathbf{D}_{T}$ and $\mathbf{D}_{F}$ given by $\mathbf{S}_{T}$ and $\mathbf{S}_{F}$ are the usual staircase triangulations of the products of simplices (see e.g. [DRS, §6.2]). 


\section{4}

In a follow-up note [KP2], we prove Cayley's theorem (Theorem 11 by an explicit volume-preserving map, mapping integer points in $\mathbf{C}_{n}$ into a simplex corresponding to integer partitions as in Theorem 1, a rare result similar in spirit to [PV]. As an application of our Theorem 2, we conclude that the volume of the convex hull of these partitions is also equal to $C_{n+1} / n$ !. While perhaps not surprising to the experts in the field $[\overline{\mathrm{Bar}}]$, the integer points in these polytopes have a completely different structure than polytopes themselves.

\section{5}

The neighbors-first search used in our construction was previously studied in [GS] in the context of the Tutte polynomial of a complete graph. Still, we find its appearance here somewhat bemusing as other graph traversal algorithms, such as depth-first search (DFS) and breadth-first search (BFS), are both more standard in algorithmic literature [Knu]. In fact, we learned that it was used in [GS] only after much of this work has been finished.

It is interesting to see what happens under graph traversal algorithms as well. In the pioneering paper [GW], Gessel and Wang showed that the identity $t^{n-1} \operatorname{Inv}_{n}(1+t)=F_{n}(t)$ can be viewed as the result of the DFS algorithm mapping connected graphs into search trees. We do not know what happens for BFS, but surprisingly the algorithm exploring edges of the graph lexicographically, from smallest to largest, also makes sense. It was shown by Crapo (in a different language, and for general matroids) to give internal and external activities [Cra]. In conclusion, let us mention that BFS, DFS and NFS are special cases of a larger class of searches known to define combinatorial bijections in a related setting [CP].

Acknowledgements. We are very grateful to Matthias Beck and Ben Braun for telling us about $[\overline{\mathrm{BBL}}]$ and the Braun Conjecture, and to Federico Ardila, Raman Sanyal and Prasad Tetali for helpful conversations. The first author was partially supported by Research Program P1-0297 of the Slovenian Research Agency. The second author was partially supported by the BSF and NSF grants.

\section{References}

[APRS] G. E. Andrews, P. Paule, A. Riese and V. Strehl, MacMahon's partition analysis. V. Bijections, recursions, and magic squares, in Algebraic Combinatorics and Applications, Springer, Berlin, 2001, 1-39.

[ABD] F. Ardila, C. Benedetti and J. Doker, Matroid polytopes and their volumes, Discrete Comput. Geom. 43 (2010), 841-854.

[Bar] A. Barvinok, Integer points in polyhedra, EMS, Zürich, 2008.

[BBL] M. Beck, B. Braun and N. Le, Mahonian partition identities via polyhedral geometry, to appear in Developments in Mathematics (memorial volume for Leon Ehrenpreis), 2011.

[Bol] B. Bollobás, Modern graph theory, Springer, New York, 1998.

[BKKS] J. Brandts, S. Korotov, M. Křížek and J. Šolc, On Nonobtuse Simplicial Partitions, SIAM Rev. 51 (2009), 317-335. 
[Cay] A. Cayley, On a problem in the partition of numbers, Philosophical Mag. 13 (1857), 245-248;

[CP] D. Chebikin and P. Pylyavskyy, A family of bijections between $G$-parking functions and spanning trees, J. Combin. Theory, Ser. A 110 (2005), 31-41.

[CL] R. Cori and Y. Le Borgne, The sand-pile model and Tutte polynomials, Adv. Appl. Math. 30 (2003), 44-52.

[CLS] S. Corteel, S. Lee and C. D. Savage, Enumeration of sequences constrained by the ratio of consecutive parts, Sém. Lothar. Combin. 54A (2007), Art. B54Aa, 12 pp.

[Cra] H. H. Crapo, The Tutte polynomial, Aeq. Math. 3 (1969), 211-229.

[DRS] J. A. De Loera, J. Rambau and F. Santos, Triangulations: Structures and Algorithms, Springer, 2008.

[Dup] J. L. Dupont, Scissors congruences, group homology and characteristic classes, World Sci., River Edge, NJ, 2001; available at http://tinyurl.com/376016

[ERS] R. Ehrenborg, M. Readdy and E. Steingrímsson, Mixed volumes and slices of the cube, $J$. Combin. Theory, Ser. A 81 (1998), 121-126.

[GGP] I. M. Gelfand, M. I. Graev and A. Postnikov, Combinatorics of hypergeometric functions associated with positive roots, in The Arnold-Gelfand mathematical seminars, Birkhäuser, Boston, MA, 1997, 205-221.

[Ges1] I. M. Gessel, A noncommutative generalization and $q$-analog of the Lagrange inversion formula, Trans. AMS 257 (1980), 455-482.

[Ges2] I. M. Gessel, Enumerative applications of a decomposition for graphs and digraphs, Discrete Math. 139 (1995), 257-271.

[GS] I. M. Gessel and B. E. Sagan, The Tutte polynomial of a graph, depth-first search, and simplicial complex partitions, El. J. Combin. 3 (1996), no. 2, RP 9, 36 pp.

[GW] I. Gessel and D. L. Wang, Depth-first search as a combinatorial correspondence, J. Combin. Theory, Ser. A 26 (1979), 308-313.

[GouJ] I. P. Goulden and D. M. Jackson, Combinatorial enumeration, John Wiley, New York, 1983.

[Had] H. Hadwiger, Ungelöste Probleme No. 13 (in German), Elem. Math. 11 (1956), 109-110.

[Hag] J. Haglund, The $q, t$-Catalan numbers and the space of diagonal harmonics, AMS, Providence, RI, 2008.

[Knu] D. E. Knuth, The Art Of Computer Programming, Vol. 1 (3rd edition), Addison-Wesley, Boston, 1997.

[KP1] M. Konvalinka and I. Pak, Triangulations of Cayley and Tutte polytopes 
[KP2] M. Konvalinka and I. Pak, A bijection for Cayley Compositions and Integer Points in Polyhedra, preprint (2011).

[LP] T. Lam and A. Postnikov, Alcoved polytopes I, Discrete Comput. Geom. 38 (2007), 453-478.

[McM] P. McMullen, The polytope algebra, Adv. Math. 78 (1989), 76-130.

[MR] C. L. Mallows and J. Riordan, The inversion enumerator for labeled trees, Bull. Amer. Math. Soc. 74 (1968), 92-94.

[Pak] I. Pak, Lectures on Discrete and Polyhedral Geometry, monograph draft; available electronically at http://www. math.ucla.edu/ pak/book.htm.

[PV] I. Pak and E. Vallejo, Combinatorics and geometry of Littlewood-Richardson cones, European J. Combin. 26 (2005), 995-1008.

[Pos] A. Postnikov, Permutohedra, associahedra, and beyond, Int. Math. Res. Not. 2009, 1026-1106.

[Sta1] R. P. Stanley, Eulerian partition of a unit hypercube, in: Higher Combinatorics, Reidel, Dordrecht, 1977; available at http://tinyurl.com/26panjb

[Sta2] R. P. Stanley, Two poset polytopes, Discrete Comput. Geom. 1 (1986), 9-23.

[Sta3] R. P. Stanley, Enumerative combinatorics, Vol. 1 and 2, Cambridge U. Press, Cambridge, $1997 / 9$.

[SP] R. P. Stanley and J. Pitman, A polytope related to empirical distributions, plane trees, parking functions, and the associahedron, Discrete Comput. Geom. 27 (2002), 603-634.

[Tut] W. T. Tutte, A contribution to the theory of chromatic polynomials, Canadian J. Math. 6 (1954), 80-91.

[Zie] G. M. Ziegler, Lectures on polytopes, Springer, New York, 1995. 